\title{
Architecture Design for Integrated Wide Area Protection and Control Systems
}

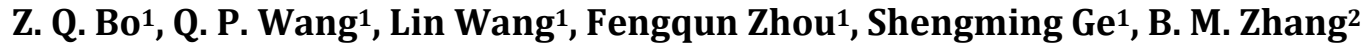 \\ ${ }^{1}$ XUJI Group, State Grid Corporation, Beijing, China \\ ${ }^{2}$ Department of Electrical Engineering, Tsinghua University, Beijing, China \\ Email: bozhiqian@263.net
}

Received December 2014

\section{Abstract}

This paper firstly reviews the recent development in power system protection and control, with special attention paid to the wide-area and integrated protection, in order to look into future development of integration of protection and control for smart grids. This paper mainly reports on the development of integrated wide area protection and control for power systems. The concept of integrated wide area protection and control is introduced, in which a hierarchical protection and control system provides the protection and control of wide area or regional power substations/ plants and their associated power network. The system is mainly divided into three levels, the local, the substation/plant and the wide area/regional protection and control. The integrated functions at each level are described in details with an aim to develop an optimal coordination mechanism between the levels. One of the core elements in the system is the synchronised wide area communication network between the substations and the protection and control system, in which latest communication technology is employed. Another important player in the system is the wide area synchronized protection and control information platform, which not only enables the fusion three line of defence for power system protection and control, but also provides a perfect tool for the application of cloud computing to substations and power networks.

\section{Keywords}

Integrated Protection, Wide Area Protection, Protection and Control, Cloud Computing

\section{Introduction}

In the late 1960's, Rockefeller [1] proposed the application of a centralized substation protection system based on a centralized computer system. The idea fits well with the concept of an overall integrated protection where the protection package would not only oversee individual units of plant but also a section of the network. However, the idea has not been widely applied until recently since the computer hardware/software and the communication technology were not available to support such an idea. Since then, relay technology has enjoyed successful development based on the application of digital techniques for power system protection. The introduction of microprocessors into protection in the 1980's generally followed the conventional approach with the implementation of distributed processing platforms that concentrated on protecting individual units of the system. 
Limited integrated protection was provided in the form of back-up protection and as such has remained a secondary function.

In recent years, the continuous development in communication technology and the possible to utilize fault generated transient for protection has resulted in the new relay protection principle to protect wide area power network [2] [3]. Immediately following the development, the concept of wide area protection mainly focused on control aspect has been presented [4]. The further development in both microprocessor and transducer technologies and the dramatic growth in signal processing power of relay platforms, and the availability of suitable communications schemes, have provided a new opportunity to revisit the concept of integrated protection. Research [5] shows that information obtained from multiple power plants and components can be used to derive new protection principles and schemes. Extensive research has been conducted on the implementation and application of integrated substation protection since. Recently the potential of the integration of protection and control for wide area application has also emerged [6].

Based on the above mentioned developments, this paper introduces a new concept of integrated wide area protection and control (IWAPC). The main focus of the concept is the integration between the protection and control, particularly at the wide area or regional level. Studies show that the IWAPC is able to provide a number of benefits to the future protection and control system with the potential to merger the three line of defense system, such as self-healing on-line decision making, in order to prevent cascading tripping of large area power network.

\section{Developments of Wide Area and Integrated Protection}

\subsection{Wide Area Protection}

In recent year, the fast development in communication technologies makes the wide area information exchange become possible. In this respect, the emergence of the wide area measurement system provides a new idea for the design of power system protection systems. The first wide area protection principle is derived from the transient based protection in 1996 [2], in which GPS time synchronization plays the major role in the design [3]. Immediately followed a summary paper in 1997 systematically outlines the concept of the so called "wide area protection" [4], which mainly focus on the control aspect of the area. The wide area protection based on novel algorithms derived from the measurements of multiple points of information is able to provide fast, reliable and accurate fault clearance, and analyzes the effects on the system stability based on the fault system analysis and takes necessary control measures, to achieve the functions of relay protection and security and stability control to prevent voltage collapse. The wide area relay protection has quickly become a hot research topic with many research results published particularly in recent years.

\subsection{Integrated Protection}

With the development of digital technology, more and more protection functions for an apparatus (line, transformer, generator etc.) have been implemented within one protective device to achieve a certain degree of integration. For example, a numeric line protection relay may have distance or current differential function as the main protection, and directional and overcurrent functions, etc. as the backup protection. The dramatic growth in signal processing power of relay platforms, and the availability of suitable communications schemes in recent years, has provided a new opportunity to derive new protection principles and schemes based on the information obtained from multiple power plants and components, which could have significant advantages over the existing protection techniques based on individual plant or component. Unlike centralized (or substation area protection) the integrated protection does not simply centralize the relay hardware/software, mainly concentrates on the developments of new concepts and algorithms based on multiple points of measurements, it is hoped from which the performance of protection can be significantly improved.

\section{Integrated Wide Area/Regional Protection and Control}

The proposed integrated wide area or regional protection and control system (IWAPC) is given in Figure 1. As shown, the latest developments in transmission system are mainly the series compensation in AC lines and high voltage DC lines; and in distribution system are mainly distributed generations and energy storage, etc. These new developments results in far more complicated characteristics than that of conventional systems, conse- 


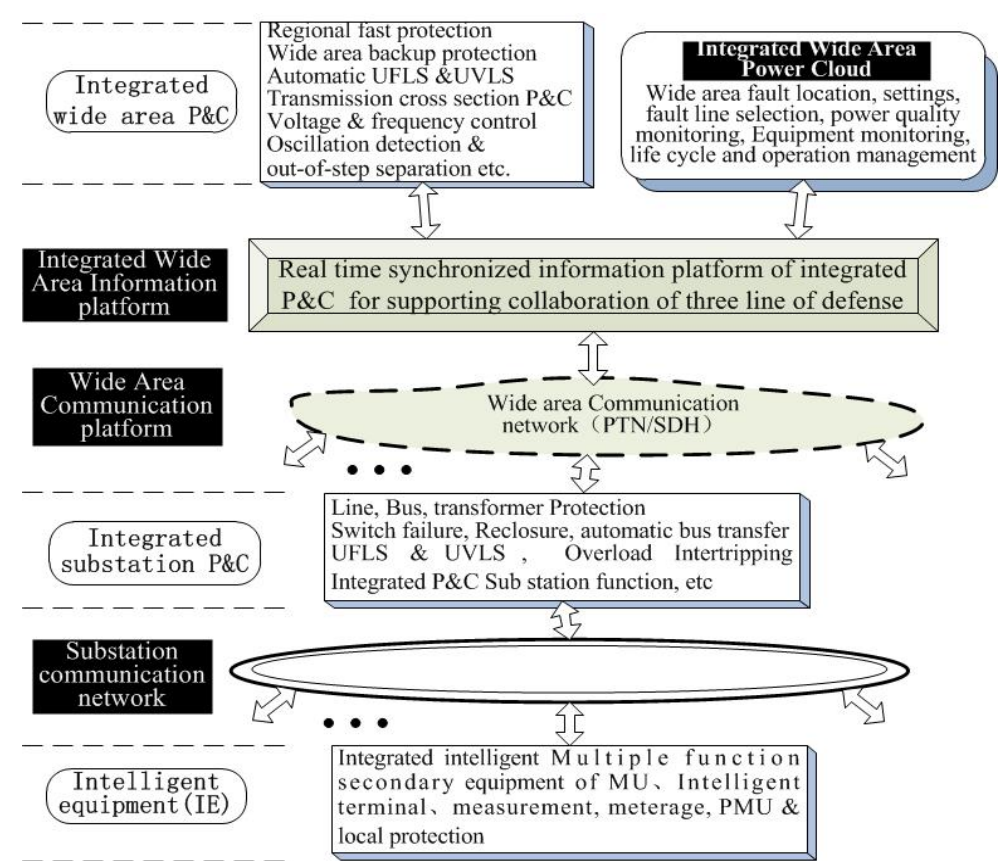

Figure 1. Integrated wide area protection and control.

quently the existing protection and control system will no longer effective to cope with the new systems, this has led to the proposed IWAPC system. As shown, the IWAPC system mainly consists of equipments at three layers: 1) the integrated multiple function intelligent equipment at the local (bay) level; 2) the integrated substation protection and control at the substation level; and 3) the integrated wide area (regional) at wide area level (IWAPC). The key parts of the system are the high speed wide area communication network (PTN) and the realtime synchronisation information platform of the IWAIP.

The IWAPC is extended to dispatching to realize the integration of dispatching automation, protection and control of power grid, and according to the three level dispatching (country, province, regional) architecture to implement the functions of regional protection, control and dispatching management.

\subsection{System Architecture}

Multiple functions intelligent equipment at the local level. As shown in Figure 1, the Intelligent equipment at local level is an integrated multiple function secondary equipment at each bay in the substation, which mainly consists of the MU, Intelligent terminal, measurement, metrology, PMU \& local protection. The IEPC is responsible to sample all real-time data and send to integrated substation P\&C and wide area P\&C, it also receives and carried out the control commands from the ISPC and IWAPC. The IEPC can be integrated into primary power apparatuses and achieve local protection for $90 \%$ of its associated line sections. It is redundant configuration with the integrated functions of fault recorder, data storage and network analysis, etc.

Integrated substation protection and control at the sunstation/plant level. The ISPC integrates functions of line, Bus, transformer protections; switch failure; autoreclosure; automatic bus transfer; UFLS, UVLS, Overload inter-tripping and substation control function, etc. It utilizes information from entire substation to achieve substation backup protection and safety automatic control etc. The CBs are used as a unit to configure the adaptive backup protection, and current differential protection is used to replace the stage overcurrent protection, breaker failure protection and dead zone protection in the conventional protection system.

Integrated wide area/regional protection and control. The IWAPC specially designed for the protection and control of transmission network is equipped with the backup protection and the IRPC for distribution network is able to offer fast protection. In addition, they both integrate functions of automatic UFLS and UVLS, voltage and frequency control, oscillation detection and out-of-step separation etc. In addition, the IWAPC also incorporates the function of transmission cross section safety P\&C. Unlike conventional protection and control, which are separated design and operation, the IWAPC integrated protection and control into one optimal com- 
bined system, which effectively coordinates the wide area (regional) protection and control, in order to achieve significant improvements in the protection and control of power systems.

\subsection{Synchronised High Speed Communication Network}

One of the most important elements of the IWAPC system is the fast communication network. The communication network proposed in here is the PTN. The present power communication network is mainly used in multiservice transport platform based on the Synchronous Digital Hierarchy (SDH). Its advantages lie in high efficiency for carrying TDM services, low latency, high reliability, with end management capabilities. However, with the new trends in smart grid development, SDH technology gradually revealed its limitations, such as low bearing efficiency and poor flexibility for data services. By contrast, PTN can realise statistical multiplexing and efficient transfer of packet service by using Packet-switched core, which can overcome the weaknesses of SDH rigid bandwidth. In addition, it can provide good quality of service, operation, administration and maintenance. Self-healing fibre optical network is employed to connect to a number of substations in the region, to ensure the fully sharing of dynamic and transient information for all electrical measurements, breaker status and protection operations, high reliability IEEE-1588 technology to ensure the synchronization timing of the sharing data, to prove the data for the integrated wide area protection and control.

\subsection{Integrated Synchronized Information Platform and Wide Area Power Cloud}

Integrated Synchronized Information Platform The real time synchronized information platform accurately correct wide area information, conduct data mining on the information to investigate the logic relation between the real-time information, to increase the sensitivity, reliability and fault tolerance capability. The real-time information is mainly divided into two types, the electrical information such as the current, voltage and angle, etc.; the state information, such as protection operation and breaker states etc. The former type can correctly reflect the fault, but requires high speed communication, in particular the time synchronization. The information can also include other types, such as the temperature of transformer oil and ambient temperature, wind speed and direction, sunshine intensity etc.

Integrated Wide Area Power Cloud [7] Based on the above mentioned information platform, a substation cloud is designed to implement functions, such as the wide area fault location, fault line selection, power quality monitoring, protection settings, etc. The extended functions also including the equipment monitoring, life cycle and operation management

Currently, many kinds of secondary equipments achieving different functions are installed in each substation, and the increasing number of distributed energy resources of small capacity is added to the system, which greatly increase the number of equipments. To implement these equipment complex functions in a specially developed "cloud" will greatly reduce the equipment investment. The cloud receives the data from the platform, which including the static, dynamic, transient measurements and states of circuit breakers etc., extracts valuable information from and allocates them to various specially designed computation algorithms in the platform to perform advanced functions in order to identify the faulted line, the accurate fault location and the contents of harmonics. The cloud computing platform, can make full use of "processing ability of cloud" to reduce the burden of terminal secondary equipment. Based on big data technique, the computing clouds enjoy strong processing power base on demand. There is no need for endlessly upgrade to improve the processing capacity of the equipment, also no need to update the software to achieve a variety of task processing. There are many more advantages can be derived from the cloud system, such as the wide area information sharing; standardization of software and algorithm; reduce in equipment investment, substation area occupation and work load for operation and maintenance. With the proposed cloud, the architecture of future substation equipment can be reshaped to provide a flexible framework for building interactive grid and improve the reliability and security of power grid.

\section{Conclusions}

This paper presents an integrated wide area protection and control system based on a hierarchical structure, which integrates protection and control at bay, substation and regional levels. Covering both transmission and distribution networks, the system is supported by the proposed high speed synchronised communication network and the real-time protection and control information platform. The system, which integrated the advanced pro- 
tection techniques and the latest developments in control system, offers not only fast protection, but also complete control of entire power network. It offers a potential for the merge the three line of defence into a unified system to more effectively ensure the reliable and safety operation of power grids.

With the continue advances in measurement, communication and information technologies, the system could have a bright future for practical application. Overall improved performance of protection and control can be expected from the proposed scheme, but for it to become useful in power system application, it is equally important that its practical implementation be readily manageable or user friendly and cost effective. The authors consider that, to achieve these goals, the totally integrated protection and control proposed offers an attractive way forward.

\section{References}

[1] Rockerfeller, G.D. (1969) Fault Protection with Digital Computer. IEEE Transactions on PAS, 88, 438-461. http://dx.doi.org/10.1109/TPAS.1969.292466

[2] Bo, Z.Q., Jayasinge, J.A.S.B., Aggarwal, R.K., et al. (1996) A New Scheme for Monitoring and Protection of Power Transmission System Based on Global Positioning System. The 31st University Power Engineering Conference, Crete, 21-24.

[3] Bo, Z.Q., Weller, G., Lomas, T., et al. (2000) Positional Protection of Transmission System Using Global Positioning System. IEEE Transactions on Power Delivery, 15, 1163-1168. http://dx.doi.org/10.1109/61.891497

[4] Ingelsson, B., Lindstrom, P.O., Karlsson, D., et al. (1997) Wide-Area Protection against Voltage Collapse. IEEE Computer Applications in Power, 10, 30-35. http://dx.doi.org/10.1109/67.625371

[5] Bo, Z.Q., He, J.H. and Dong, X.Z. (2005) Integrated Protection of Power Network. Relay, 33, 33-41

[6] Bo, Z.Q., Zhang, B.H., Dong, X.Z., He, J.H., et al. (2013) The Development of Protection Intellectuation and Smart Relay Network. Power System Protection and Control, 41, 1-12.

[7] Bo, Z.Q., Wang, L., Zhou, F.Q., et al. (2014) Substation Cloud Computing for Secondary Auxiliary Equipment. Powercon 2014, Chengdu, October 2014. 